\title{
MRI Image Segmentation Using Conditional Spatial FCM Based on Kernel-Induced Distance Measure
}

\author{
Babak Gharnali \\ Department of Computer Engineering \\ Islamic Azad University, Buinzahra Branch \\ Buinzahra, Iran \\ babakgharnali.ai@gmail.com
}

\author{
Siavash Alipour \\ Department of Electrical and Electronic Engineering \\ Malek-Ashtar University of Technology \\ Tehran, Iran \\ siavash.alipur@gmail.com
}

\begin{abstract}
Fuzzy C-means (FCM) clustering is the widest spread clustering approach for medical image segmentation because of its robust characteristics for data classification. But, it does not fully utilize the spatial information and is therefore very sensitive to noise and intensity inhomogeneity in magnetic resonance imaging (MRI). In this paper, we propose a conditional spatial kernel fuzzy C-means (CSKFCM) clustering algorithm to overcome the mentioned problem. The approach consists of two successive stages. First stage is achieved through the incorporation of local spatial interaction among adjacent pixels in the fuzzy membership function imposed by an auxiliary variable associated with each pixel. The variable describes the involvement level of each pixel for construction of membership functions and different clusters. Then, we adapted a kernelinduced distance to replace the original Euclidean distance in the FCM, which is shown to be more robust than FCM. The problem of sensitivity to noise and intensity inhomogeneity in MRI data is effectively reduced by incorporating a kernel-induced distance metric and local spatial information into a weighted membership function. The experimental results show that the proposed algorithm has advantages in accuracy and robustness against noise in comparison with the FCM, SFCM and CSFCM methods on MRI brain images.
\end{abstract}

Keywords-image segmentation; MRI; fuzzy C-means; noise

\section{INTRODUCTION}

Segmentation is an essential preprocessing step in computer-guided medical image analysis and diagnosis [1]. It helps doctors extract soft tissue regions from the respective organs of the human body for surgical decisions, abnormality detection and therapy management. MRI is one of the most eminent medical imaging techniques and segmentation is a critical stage in the investigation of MRI images. Some factors complicate segmentation in medical images such as noise, normal anatomic variation, post-surgical anatomic variation, vague and incomplete boundaries, variation of contrast, inhomogeneities in the boundaries of the object of interest, motion blurring artifacts and so on [2]. To address these difficulties, clustering methods have been extensively studied and widely used in MIS, with promising results [3]. Among the different clustering methods devised in the past for medical images, the fuzzy c-means (FCM) algorithm has proved its efficacy. Unlike hard clustering methods, like k-means algorithm, which assign pixels exclusively to one cluster, the FCM algorithm allows pixels to have relation with multiple clusters with varying degree of memberships. The main disadvantage of the FCM is that it is sensitive to noise and inhomogeneity which leads to erroneous segmentation results and undesired visual quality in case of MRI brain image data. To overcome the problems of traditional FCM algorithm, many researchers modified the objective function by considering different criteria and presented more efficient and robust clustering algorithms [4-8].

Authors in [9] introduced a class of robust non-Euclidean distance measure for the objective function to enhance the robustness of the original FCM clustering algorithm and to reduce noise and outliers. Enhanced FCM is proposed in [10] to fasten the segmentation process as well as to reduce the noise effect. To deal with the inhomogeneity problem, many algorithms have been proposed by adding correction steps before image segmenting [11,12] or by modeling the image as the product of the original image and a smooth varying multiplier field [13,14]. In [15], authors introduced a novel energy term based on multilayer structure of local intensity information to segment images with intensity inhomogeneity. Authors in [16] proposed a region-based method using a local similarity factor to enhance the performance in segmenting images with intensity inhomogeneity and noise. Authors in [7] proposed a conditional spatial fuzzy C-means clustering algorithm (CSFCM) to address the FCM problem. The CSFCM algorithm incorporates conditioning effects, imposed by some auxiliary (conditional) variables, as well as spatial information into the final membership function to improve robustness to the noise and inhomogeneity, providing thereby superior segmentation results.

In this paper, we propose a conditional spatial kernel fuzzy C-means (CSKFCM) clustering algorithm that can effectively segment MRI brain images with the presence of noise and intensity inhomogeneity. The proposed algorithm incorporates a kernel-induced distance to replace the original Euclidean distance in CSFCM algorithm which leads to better results. It is shown that the proposed algorithm has better segmentation results on simulated or real MR images corrupted by noise and other artifacts than the standard algorithms such as CSFCM. The rest of the paper is organized as follows: in section II, we 
briefly introduce the CSKFCM method to prepare the description of our proposed segmentation framework which is explained in Section III in detail. The experimental results and conclusions are presented in Sections IV and V respectively.

\section{BACKGROUND}

FCM clustering [17-19] is an unsupervised technique that has been successfully applied to feature analysis, clustering, and classifier designs in fields such as astronomy, geology, medical imaging, target recognition, and image segmentation. The FCM algorithm, where the correlation between the neighboring pixels is not considered, fails to generate accurate clusters. Authors in [7] proposed a conditional spatial fuzzy cmeans algorithm (CSFCM) to address this issue. This mechanism ensures that if the neighboring pixels share similar characteristics, the center pixel should have higher probability of being grouped to the same cluster as the neighboring pixels. Thus, the algorithm takes the level of involvement of a pixel into a cluster by considering its neighborhood. A local membership function is defined as follows:

$$
\begin{aligned}
U_{i k} & =\frac{f_{i k}}{\left\|\left(x_{i}\right)-\left(v_{i}\right)\right\|^{\frac{2}{m-1}} / \sum_{c=1}^{C}\left\|\left(x_{k}\right)-\left(v_{c}\right)\right\|^{\frac{2}{m-1}}} \\
f_{i k} & =\frac{\left.\sum_{j \in N\left(x_{k}\right)}\right)_{i k}}{M}
\end{aligned}
$$

Where $f_{i k}$ is the conditioning variable which defines the level of involvement of pixel $X_{i}$ into the $i_{t h}$ cluster by considering its neighborhood in a spatial domain. $N\left(x_{k}\right)$ is the square box having the pixel $X_{i}$ as its center and $M$ is the number of pixels in the box. The membership value function of the CSFCM is the combination of the global membership values $\mu_{i k}$ and the local membership values $U_{i k}$ as following:

$$
\begin{aligned}
Z_{i k} & =\frac{\left(\mu_{i k}\right)^{p} U_{i k} q}{\sum_{c=1}^{C}\left(\mu_{c k}\right)^{p}\left(U_{c k}\right)^{q}} \\
W_{i} & =\frac{\sum_{k=1}^{N} z_{i k}^{m} x_{k}}{\sum_{k=1}^{N} z_{i k}^{m}}
\end{aligned}
$$

where $\mathrm{p}$ and $\mathrm{q}$ are the parameters to control the relative importance of both global and local membership functions. Figure 1 displays the full explanation of the CSFCM method.

\section{PROPOSED ALGORITHM}

The CSFCM algorithm employs squared-norm in similarity measurement which is effective in grouping "spherical" clusters. In fact, due to the use of nonrobust Euclidean distance, it leads to nonrobust results. In this paper, based on the recognized power of the "kernel method" [20, 21] in recent machine learning community, we present a kernelized CSFCM algorithm to address this issue. Unlike the CSFCM algorithm which adopts the squared-norm distance metric in the objective function, the proposed algorithm uses a kernel-induced metric and is shown to be more robust to noise and outlier than classical algorithms. Here, we adapted a kernel-induced distance to replace the original Euclidean distance in the CSFCM. By replacing the inner product with an appropriate "kernel" function, one can implicitly perform a nonlinear mapping to a higher-dimensional feature space without increasing the number of parameters. The philosophy of the "kernel method" is that every (linear) algorithm that only uses scalar products can be extended to the corresponding (nonlinear) version of this algorithm which is implicitly executed in a higher feature space through kernels [22].

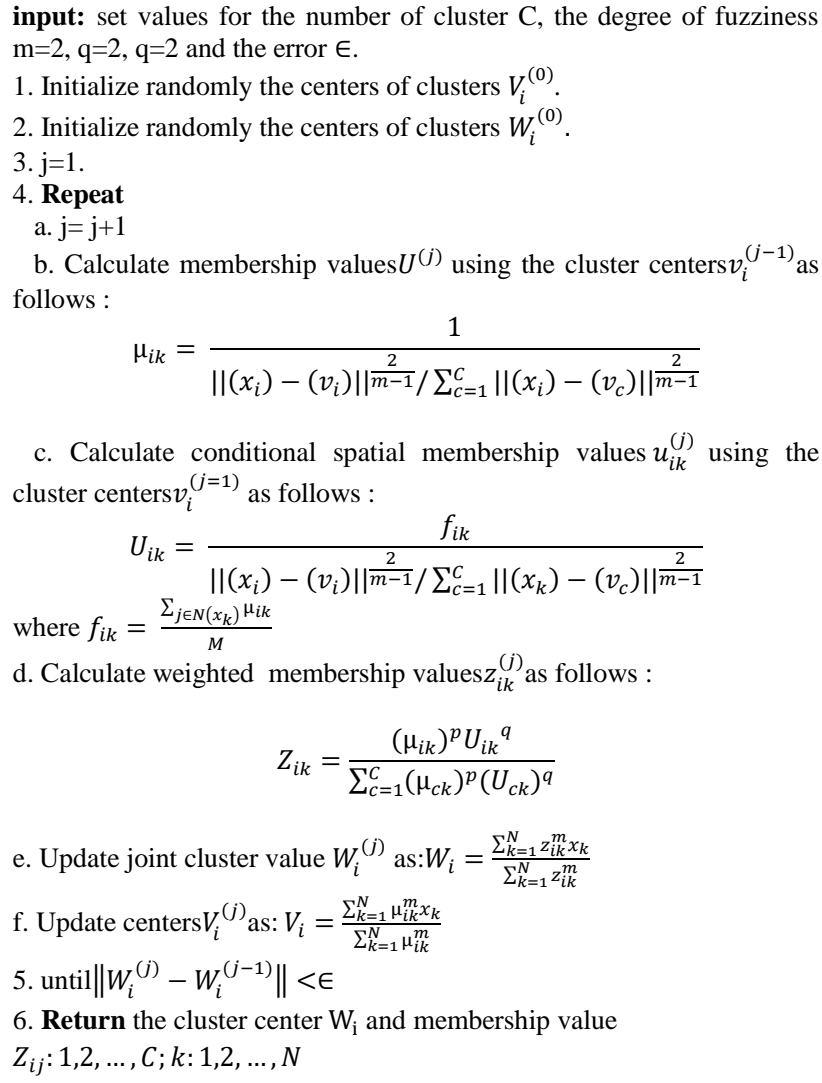$$
\mu_{i k}=\frac{1}{\left\|\left(x_{i}\right)-\left(v_{i}\right)\right\|^{\frac{2}{m-1}} / \sum_{c=1}^{C}\left\|\left(x_{i}\right)-\left(v_{c}\right)\right\|^{\frac{2}{m-1}}}
$$

c. Calculate conditional spatial membership values $u_{i k}^{(j)}$ using the cluster centers $v_{i}^{(j=1)}$ as follows :

$$
U_{i k}=\frac{f_{i k}}{\left\|\left(x_{i}\right)-\left(v_{i}\right)\right\|^{\frac{2}{m-1}} / \sum_{c=1}^{C}\left\|\left(x_{k}\right)-\left(v_{c}\right)\right\|^{\frac{2}{m-1}}}
$$

where $f_{i k}=\frac{\sum_{j \in N\left(x_{k}\right)} \mu_{i k}}{M}$

d. Calculate weighted membership values $z_{i k}^{(j)}$ as follows :

$$
Z_{i k}=\frac{\left(\mu_{i k}\right)^{p} U_{i k}^{q}}{\sum_{c=1}^{C}\left(\mu_{c k}\right)^{p}\left(U_{c k}\right)^{q}}
$$

e. Update joint cluster value $W_{i}^{(j)}$ as: $W_{i}=\frac{\sum_{k=1}^{N} z_{i k}^{m} x_{k}}{\sum_{k=1}^{N} z_{i k}^{m}}$

f. Update centers $V_{i}^{(j)}$ as: $V_{i}=\frac{\sum_{k=1}^{N} \mu_{i k}^{m} x_{k}}{\sum_{k=1}^{N} \mu_{i k}^{m}}$

5. until $\left\|W_{i}^{(j)}-W_{i}^{(j-1)}\right\|<\epsilon$

6. Return the cluster center $\mathrm{W}_{\mathrm{i}}$ and membership value

$Z_{i j}: 1,2, \ldots, C ; k: 1,2, \ldots, N$

\section{Fig. 1. The CSFCM Algorithm}

A kernel in the feature space can be represented as a function $\mathrm{K}$ below:

$$
K(x, y)=\langle\Phi(x), \Phi(y)\rangle
$$

where $\Phi$ stands as implicit nonlinear map, and $\langle\Phi(x), \Phi(y)\rangle$ denotes the inner product operation.

In the CSFCM objective function, we express the distance function using inner product space. By constraining ourselves to the Euclidean distance in $\left\|\Phi\left(x_{k}\right)-\Phi\left(v_{i}\right)\right\|$ the squared distance is computed in the kernel space using a kernel function. Here we use only the Gaussian RBF kernel for simplicity. So, if we employ the nonlinear map with the Gaussian kernel substitution in the CSFCM objective function, the global and local membership function of CSFCM can be rewritten as follows:

$\mu_{i k}=\frac{1}{\left(1-\mathrm{K}\left(x_{k}, v_{i}\right)\right)^{\frac{2}{m-1}} / \sum_{C=1}^{C}\left(1-\mathrm{K}\left(x_{k}, v_{c}\right)\right)^{\frac{2}{m-1}}}$ 


$$
U_{i k}=\frac{f_{i k}}{\left(1-\mathrm{K}\left(x_{k}, v_{i}\right)\right)^{\frac{2}{m-1}} / \sum_{c=1}^{C}\left(1-\mathrm{K}\left(x_{k}, v_{i}\right)\right)^{\frac{2}{m-1}}}
$$

Where $K(x, y)=\Phi(x)^{T} \Phi(y)$ is an inner product Gaussian RBF kernel function, and $K(x, y)=\exp \left[-\left(\|x-y\|^{2}\right) / 2 \sigma^{2}\right]$. Moreover, cluster centers can be rewritten as follow:

$$
v_{i}=\frac{\sum_{k=1}^{n} u_{i k}^{m} K\left(x_{k}, v_{i}\right) x_{k}}{\sum_{k=1}^{n} u_{i k}^{m} K\left(x_{k}, v_{i}\right)}
$$

According to (6)-(8), the data point $x_{k}$ is endowed with an additional weight $K\left(x_{k}, v_{i}\right)$, which measures the similarity between $x_{k}$ and $v_{i}$ based on their effects with respect to the pixels' distance. It means that the kernel function gives more weight to nearer pixels and vice versa. The proposed CSKFCM algorithm is be summarized in the Figure 2.

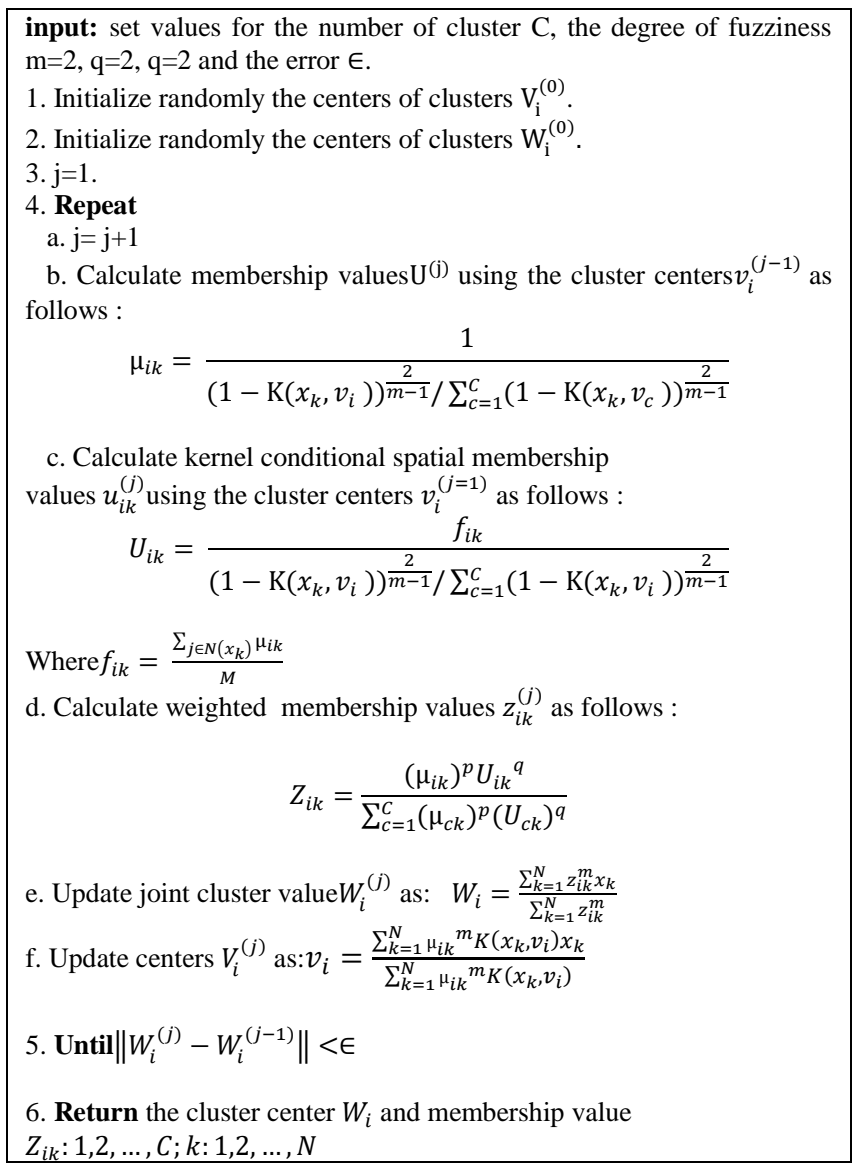

Fig. 2. The Proposed CSKFCM Algorithm

\section{EXPERIMENTAL RESULTS}

This paper performs two groups of experiments to evaluate the proposed algorithm qualitatively and quantitatively. The qualitative evaluation compares the output of the proposed algorithm with the following algorithms: FCM [23], SFCM [24], and CSFCM [7]. The quantitative evaluation shows the final output with the reference segmented image and compares the segmentation results of the proposed method with three fuzzy-based mentioned algorithms along the same line based on ground-truth images. The quantitative evaluation is computed based on Dice criteria [25,26]. The Dice factor which is common in image segmentation has been measured for segmentation algorithms as a quantitative evaluation. The experiments and performance evaluation were carried out on three different datasets. One is a simple synthetic image, another is the classical simulated brain database borrowed from McConnell Brain Imaging Centre of the Montreal Neurological Institute, McGill University [27], and the last one is an MR Image borrowed from [2]. The original images are stored in gray scale space. These images are commonly used in papers [28, 29-34] for image segmentation purposes and the algorithms compared have employed these images in their experiments. Next, the setting of parameters, the comparison of algorithms and the evaluation of each performance are explained in detail.

\section{A. Setting Experimental Parameters}

The max iteration, $t_{\max }$, was considered 200 . The parameters in the FCM based methods were set as follows: (i) The weighting exponent was set to be $m=2$ since it had been shown to be the most effective one [35]. (ii) The termination criteria was set to be $\varepsilon=0.01$ since most reports showed good success with $\varepsilon \in[0.01,0.0001]$ [ 36 ]. (iii) The Gaussian RBF kernel width as proposed method parameter was set to be $\sigma=150$ since it showed better results than other $\sigma$ values [36]. Moreover, the parameters in the CSFCM [7] method were set as follows: (i) The parameters of weighted membership function were set to be $p=2$ and $q=2$ since it had been shown that the algorithm provides superior results using these values [CSFCM]. (ii) The size of the neighborhood was set to $N\left(x_{k}\right)=9$ $(3 \times 3$ window centered around each pixel).

\section{B. Qualitative Evaluation}

The synthetic image is shown in Figure 3(a). It contains a two-class pattern corrupted by Gaussian noise with mean $=0$, variance $=0.02$. Figures $3(\mathrm{~b})-(\mathrm{e})$ show the segmentation results of FCM, SFCM, CSFCM and the proposed CSKFCM respectively. As shown in Figure 3(b), without spatial constraints, the FCM cannot separate the two classes. Although, the SFCM and CSFCM employ the correlation between the neighboring pixels, they fail to generate accurate clusters as shown in Figures 3(c) and (d). By comparing Figure 3(d) with the others, we can see that the proposed CSKFCM method provides a better segmentation result than its counterparts. Note that because of the injection of the kernel, CSKFCM needs more execution time than CSFCM, and correspondingly, CSKFCM is slower than CSFCM. Typically, the algorithms without kernel are several times faster than those with injection of kernels.

The next experiment is designed to evaluate the performance of the proposed method on a T1-weighted simulated brain image with the presence of noise and intensity inhomogeneity. The target is to segment different tissue regions: cerebrospinal fluid (CSF), grey matter (GM) and white matter (WM). Figure 4 shows the segmentation results of a T1weighted MR image (slice 100, Figure 4(a) with 7\% noise and $20 \%$ inhomogeneity. Figure 4(b)-(d) shows the segmented regions of the CSF, GM and WM, respectively by the FCM 
algorithm. Figures 4(e)-(g) show the segmented regions of the CSF, GM and WM, respectively by the SFCM algorithm. Figures 4(h)-(j) show the segmented regions of the CSF, GM and WM, respectively by the CSFCM algorithm. Figures 4(k)(m) show the segmented regions of the CSF, GM and WM, respectively by the proposed CSKFCM algorithm.
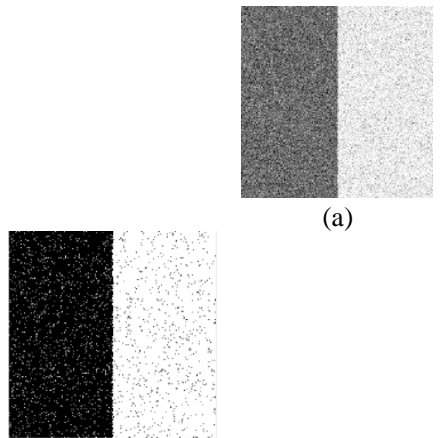

(a)

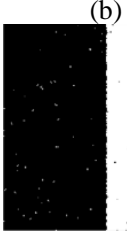

(d)

(e)

Fig. 3. Comparison of segmentation results on a synthetic image corrupted by Gaussian noise with variance $=0.02$. (a) The original image, (b) using FCM, (c) using SFCM, (d) using CSFCM, and (e) using CSKFCM

The last row of Figure 4 shows the result of the proposed algorithm. It can be seen that this algorithm performs well for the MRI brain image with the presence of noise and intensity inhomogeneity. However, the rest fails in most situations and also several artifacts are present in the segmented regions of the CSF, GM and WM. The reason is that the CSKFCM attempts to unify the intensity and spatial information as a whole and it employs a kernel-induced new metric to replace the Euclidean norm in the original space. Consequently, it shows less susceptibility to noise.

In the last experiment of this section, we tested the performance of the proposed CSKFCM algorithm on another MRI image data from [2] corrupted by Gaussian noise with mean $=0$, variance $=0.003$ as shown in Figure 5(a). The second to fifth rows of Figure 5 show segmentation results by FCM, SFCM, CSFCM, and CSKFCM respectively. By comparing them, we can see that the proposed method provides a better segmentation result than its counterparts.

\section{Qualitative Evaluation}

To evaluate the results of Brain MRI mage segmentation, it is necessary to make a quantitative evaluation of different algorithms. The comparisons are illustrated using the Dice Similarity coefficient (DSC) $[11,23]$ as a similarity criterion, which is defined by:

$$
\operatorname{DSC}\left(\Omega_{\mathrm{s}}, \Omega_{\mathrm{r}}\right)=\frac{2 \operatorname{Area}\left(\Omega_{\mathrm{s}} \cap \Omega_{\mathrm{r}}\right)}{\left(\operatorname{Area}\left(\Omega_{\mathrm{s}}\right)+\operatorname{Area}\left(\Omega_{\mathrm{r}}\right)\right)}
$$

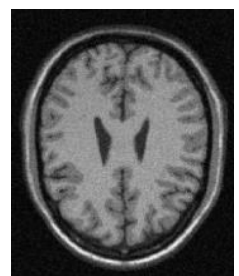

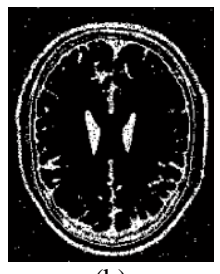

(b)

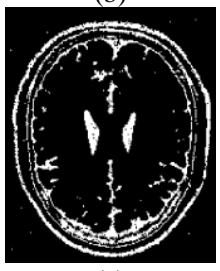

(e)

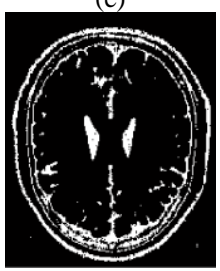

(h)

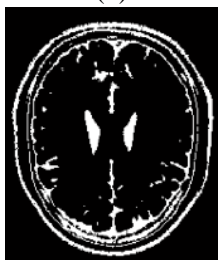

(k)

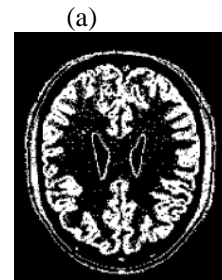

(c)
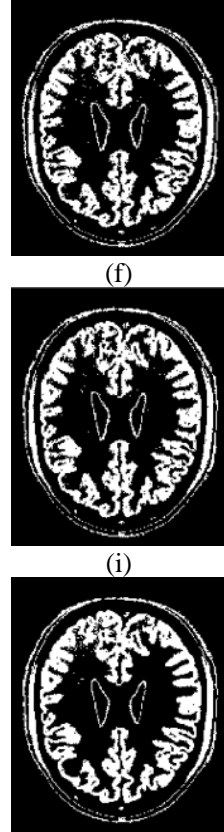

(1)

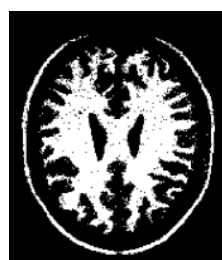

(d)

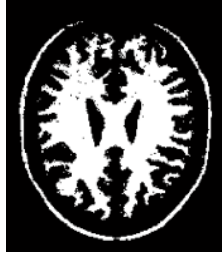

(g)

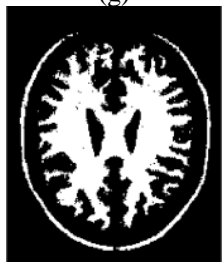

(j)

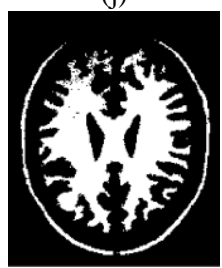

(m)
Fig. 4. Segmentation results of the CSF, GM and WM (from left to right) by the different algorithms on a T1-weighted MRI brain image with $7 \%$ noise and $20 \%$ inhomogeneity (a). (b)-(d): FCM algorithm; (e)-(g): SFCM algorithm; (h)-(j): CSFCM algorithm and (k)-(m): the proposed CSKFCM algorithm.

In (9), $\Omega_{s}$ is the final segmented region and $\Omega_{r}$ is the corresponding reference region. This coefficient varies from 0 to 1 and measures the degree of agreement between the two regions. It is 1 if the two regions are identical and 0 when they are completely different. Table I gives the segmentation accuracy of four methods in Figure 3(a) based on ground-truth image in Figure 6(a), where the segmentation accuracy is computed using the Dice similarity coefficient. Table II shows the results of applying the Dice similarity coefficient for three segmented parts of the simulated MRI brain image in Figures 2(a): CSF, GM and WM using the FCM, SFCM, CSFCM and the proposed method. The second row of Figure 6 shows the reference or the region of interest (ROI) of the CSF, GM and WM regions of the brain image in Figure 4(a). Table III shows the results of applying the Dice similarity coefficient for the WM segmented parts of the MRI brain image in Figure 3(a) 
using the FCM, SFCM, CSFCM and the proposed method. Figure 4(e) shows the reference of the WM region of the brain image in Figure 5(a). From Tables I-III, it can be seen that the best results in term of the Dice coefficient (highest value) are achieved by our proposed method. In addition, by comparing the CSFCM and the proposed CSKFCM results from the Tables, we can see the advantages in using the kernel-induced metric to replace the original Euclidean norm metric in CSFCM objective function.

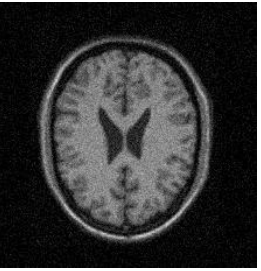

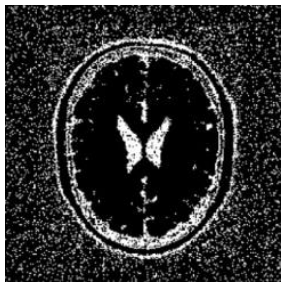

(b)

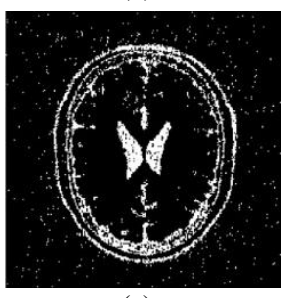

(e)

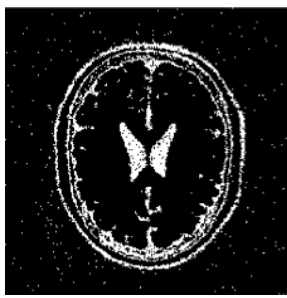

(h)

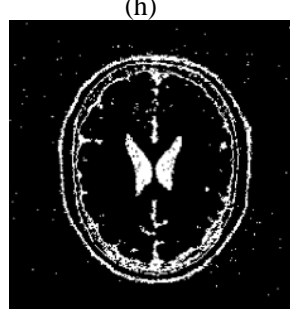

(k)

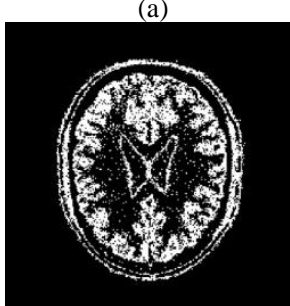

(c)
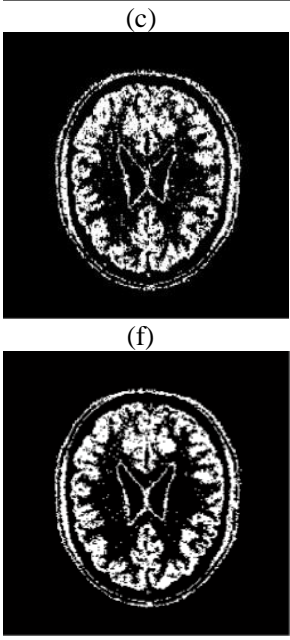

(i)

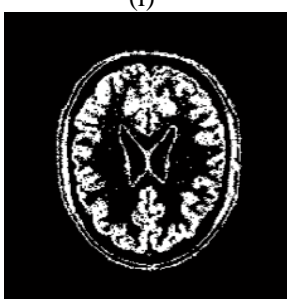

(1)

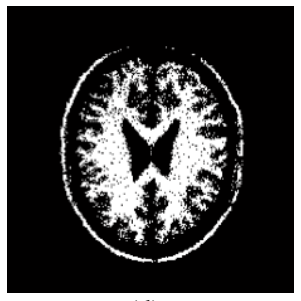

(d)

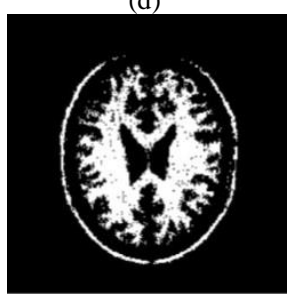

(g)

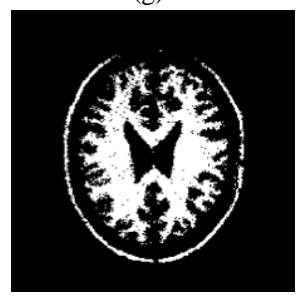

(j)

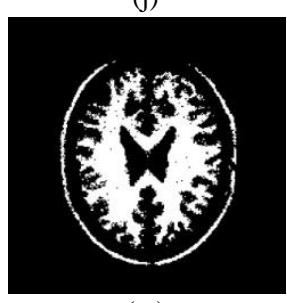

(m)
Fig. 5. Segmentation results of the CSF, GM and WM (from left to right) by the different algorithms on an MRI brain image contaminated with Gaussian noise (mean $=0$ and variance $=0.003)(a)$. (b) $-(d)$ : FCM algorithm. (e) $-(\mathrm{g})$ : SFCM algorithm. (h)-(j): CSFCM algorithm. (k)-(m): Proposed CSKFCM algorithm.

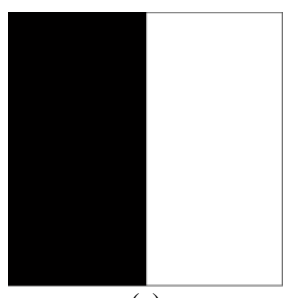

(a)

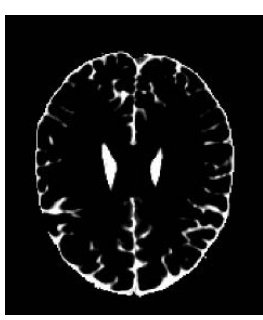

(b)

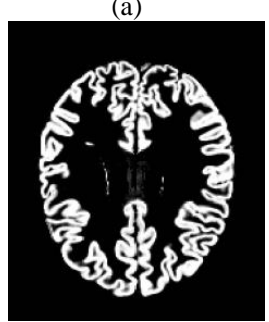

(c)

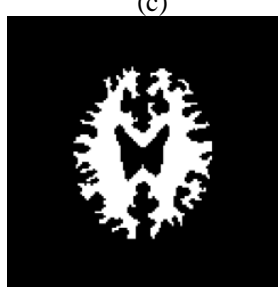

(e)

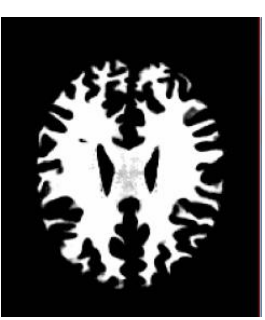

(d)
Fig. 6. Ground-truth segmentation of images. a ROI of the synthetic image in Figure 4(a). (b)-(d): CSF, GM and WM ROI of the simulated brain image in Figure 4(a). (e): WM ROI of the brain image in Figure 5(a)

TABLE I. COMPUTATION DICE SIMILARITY COEFFICIENTS (FIGURE 3(A))

\begin{tabular}{|c|c|c|c|c|}
\hline & FCM & SFCM & CSFCM & Proposed method \\
\hline Figure. 1a & 0.9560 & 0.9856 & 0.9904 & 0.9982 \\
\hline
\end{tabular}

TABLE II. COMPUTATION DICE SIMILARITY COEFFICIENTS (FIGURE 4(A))

\begin{tabular}{|c|c|c|c|c|}
\hline & FCM & SFCM & CSFCM & Proposed method \\
\hline CSF & 0.9560 & 0.9856 & 0.9904 & 0.9982 \\
\hline GM & 0.8423 & 0.8623 & 0.8930 & 0.9086 \\
\hline WM & 0.8621 & 0.8876 & 0.9030 & 0.9191 \\
\hline
\end{tabular}

TABLE III. COMPUTATION DICE SIMILARITY COEFFICIENTS (FIGURE 5(A))

\begin{tabular}{|c|c|c|c|c|}
\hline & FCM & SFCM & CSFCM & Proposed method \\
\hline WM & 0.8015 & 0.8230 & 0.8446 & 0.8594 \\
\hline
\end{tabular}

\section{CONCLUSION}

In this paper, a new approach for segmentation of corrupted by noise MRI images has been presented. The proposed algorithm is based on incorporating the kernel method and the spatial information into the final membership function of the FCM to effectively segment MR images corrupted by noise and inhomogeneity. We assessed the effectiveness of the proposed algorithm, qualitatively and quantitatively, on synthetic and MR images available in segmentation data-bases that are commonly used in papers. In the qualitative evaluation, the output of the proposed algorithm was compared with the FCM, SFCM and CSFCM methods in noisy cases. The quantitative evaluation was calculated based on the Dice factor. Experimental results demonstrate that the proposed algorithm produces better results by successfully detecting the object 
boundaries and also is more robust to Gaussian noise than other fuzzy clustering algorithms in segmenting MRI images.

\section{REFERENCES}

[1] J. S. Duncan, N. Ayache, "Medical image analysis: Progress over two decades and the challenges ahead", IEEE Transactions on Pattern Analysis and Machine Intelligence, Vol. 22, No. 1, pp. 85-106, 2000

[2] T. Dietenbeck, M. Alessandrini, D. Friboulet, O. Bernard, "CREASEG: a free software for the evaluation of image segmentation algorithms based on level-set”, 2010 17th IEEE International Conference on Image Processing (ICIP), Hong Kong, China, Vol. 1, No. 1, pp. 665-668, September 26-29, 2010

[3] C. Xu, D. Pham, J. Prince, Image segmentation using deformable models. Handbook of medical imaging, Elsevier, 2000

[4] A. W. C. Liew, S. H. Leung, W. H. Lau, "Fuzzy image clustering incorporating spatial continuity", IEE Proceedings-Vision, Image and Signal Processing, Vol. 147, No. 2, pp. 185-192, 2000

[5] D. L. Pham, "Spatial models for fuzzy clustering. Computer vision and image understanding", Computer Vision and Image Understanding, Vol. 84, No. 2, pp. 285-297, 2001

[6] A. W. Liew, H. Yan, "An adaptive spatial fuzzy clustering algorithm for 3-D MR image segmentation”, IEEE Transactions on Medical Imaging, Vol. 22, No. 9, pp. 1063-1075, 2003

[7] S. K. Adhikari, J. K. Sing, D. K. Basu, M. Nasipuri, "Conditional spatial fuzzy C-means clustering algorithm for segmentation of MRI images", Applied Soft Computing, Vol. 34, pp.758-769, 2015

[8] J. K. Sing, S. K. Adhikari, D. K. Basu, "A modified fuzzy C-means algorithm using scale control spatial information for MRI image segmentation in the presence of noise", Journal of Chemometrics, Vol. 29, No. 9, pp. 492-505, 2015

[9] S. R. Kannan, R. Devi, S. Ramathilagam, K. Takezawa, "Effective FCM noise clustering algorithms in medical images", Computers in biology and medicine, Vol. 43, No. 2, pp. 73-83, 2013

[10] L. Szilagyi, Z. Benyo, S. M. Szilágyi, H. Adam, "MR brain image segmentation using an enhanced fuzzy c-means algorithm", Proceedings of the 25th Annual International Conference of the IEEE Engineering in Medicine and Biology Society, pp. 724-726, Cancun, Mexico, September 17-21, 2003

[11] B. M. Dawant, A. P. Zijdenbos, R. A. Margolin "Correction of intensity variations in MR images for computer-aided tissue classification", IEEE Transactions on Medical Imaging, Vol. 12, No, 4, pp. 770-781, 1993

[12] B. Johnston, M. S. Atkins, B. Mackiewich, M. Anderson, "Segmentation of multiple sclerosis lesions in intensity corrected multispectral MRI", IEEE Transactions on Medical Imaging, Vol. 15, No. 2, pp. 154-169, 1996

[13] W. M. Wells, W. L. Grimson, R. Kikinis, F. A. Jolesz, "Adaptive segmentation of MRI data", IEEE Transactions on Medical Imaging, Vol. 15, No. 4, pp. 429-442, 1996

[14] D. L. Pham, J. Prince, "An adaptive fuzzy C-means algorithm for image segmentation in the presence of intensity inhomogeneities", Pattern Recognition Letters, Vol. 20, No. 1, pp. 57-68, 1999

[15] H. Min, X.-F. Wang, D.-S. Huang, W. Jia, “A novel dual minimization based level set method for image segmentation", Neurocomputing, Vol. 214, pp. 910-926, 2016

[16] S. Niu, Q. Chen, L. de Sisternes, Z. Ji, Z. Zhou, D. Rubin, "Robust noise region-based active contour model via local similarity factor for image segmentation", Pattern Recognition, Vol. 61, pp. 104-119, 2017

[17] J. Bezdek, L. Hall, L. Clarke, "Review of MR image segmentation techniques using pattern recognition", Medical physics, Vol. 20, No. 4, pp. 1033-1048, 1993

[18] N. Iyer, A. Kandel, M. Schneider, "Feature-based fuzzy classification for interpretation of mammograms", Fuzzy Sets and Systems, Vol. 114, No. 2, pp. 271-280, 2000

[19] M. S. Yang, Y. J. Hu, K. C. Lin, C. C. Lin, "Segmentation techniques for tissue differentiation in MRI of ophthalmology using fuzzy clustering algorithms", Magnetic Resonance Imaging, Vol. 20, No. 2, pp. 173-179, 2002

[20] K.-R. Muller, S. Mika, G. Ratsch, K. Tsuda, B. Scholkopf, "An introduction to kernel-based learning algorithms", IEEE Transactions on Neural Networks, Vol. 12, No. 2, pp. 181-201, 2001

[21] N. Cristianini, J. Shawe-Taylor, An introduction to support vector machines and other kernel-based learning methods, Cambridge University Press, 2000

[22] D.-Q. Zhang, S.-C. Chen, "Kernel-based fuzzy and possibilistic c-means clustering", Proceedings of the International Conference Artificial Neural Network, Vol. 122, pp. 122-125, 2003

[23] J. Bezdek, Pattern Recognition with Fuzzy Objective Function Algorithms, Kluwer Academic Publishers, 1983

[24] K. S. Chuang, H. L. Tzeng, S. Chen, J. Wu, T. J. Chen, "Fuzzy c-means clustering with spatial information for image segmentation. computerized medical imaging and graphics", Vol. 30, No. 1, pp. 9-15, 2006

[25] Y. Shi, W. C. Karl, "A real-time algorithm for the approximation of level-set-based curve evolution", IEEE Transactions on Image Processing, Vol. 17, No. 5, pp. 645-656, 2008

[26] L. R. Dice, "Measures of the amount of ecologic association between species", Ecology, Vol. 26, No. 3, pp. 297-302, 1945

[27] C. A. Cocosco, V. Kollokian, R. K.-S. Kwan, G. Bruce Pike, A. C. Evans, "Brainweb: Online interface to a 3D MRI simulated brain database", Neuro Image, 1997

[28] T. F. Chan, L. A. Vese, "Active contours without edges". IEEE Transactions on Image Processing, Vol. 10, No. 2, pp. 266-277, 2001

[29] O. Bernard, D. Friboulet, P. Thévenaz, M. Unser, "Variational B-spline level-set: a linear filtering approach for fast deformable model evolution", IEEE Transactions on Image Processing, Vol. 18, No. 6, 1179-1191, 2009

[30] E .Nadernejad, S. Sharifzadeh, "A new method for image segmentation based on Fuzzy C-means algorithm on pixonal images formed by bilateral filtering", Signal, Image and Video Processing, Vol. 7, No. 5, pp. 855-863, 2013

[31] S. Hu, E. A. Hoffman, J. M. Reinhardt, "Automatic lung segmentation for accurate quantitation of volumetric X-ray CT images", IEEE Transactions on Medical Imaging, Vol. 20, No. 6, pp. 490-498, 2001

[32] R. Chan, H. Yang, T. Zeng, "A two-stage image segmentation method for blurry images with Poisson or multiplicative gamma noise", SIAM Journal on Imaging Sciences, Vol. 7, No. 1, pp. 98-127, 2014

[33] M. Droske, B. Meyer, M. Rumpf, C. Schaller, "An adaptive level set method for medical image segmentation", Lecture Notes in Computer Science, Vol. 2082, pp. 416-422, Springer, Berlin, Heidelberg, 2001

[34] C. Li, C.-Y. Kao, J. C. Gore, Z. Ding, "Minimization of region-scalable fitting energy for image segmentation", IEEE Transactions on Image Processing, Vol. 17, No. 10, pp. 1940-1949, 2008

[35] J. C. Bezdek, J. Keller, R. Krisnapuram, N. Pal, Fuzzy models and algorithms for pattern recognition and image processing, Springer Science \& Business Media, Vol. 4, 1999

[36] D. -Q. Zhang, S.-C. Chen, "A novel kernelized fuzzy c-means algorithm with application in medical image segmentation", Artificial Intelligence in Medicine, Vol. 32, No. 1, pp. 37-50, 2004 